\title{
Optimizing potentiometric ionophore and electrode design for environmental on-site control of antibiotic drugs: Application to sulfamethoxazole
}

\author{
S.A.A. Almeida, Liliana A.A.N.A. Truta, Raquel B. Queirós, M.C.B.S.M. Montenegro, \\ Alexandre L. Cunha, M.G.F. Sales
}

\section{ABSTRACT}

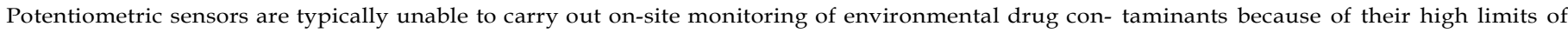

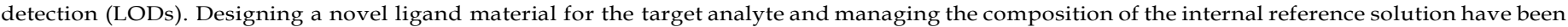

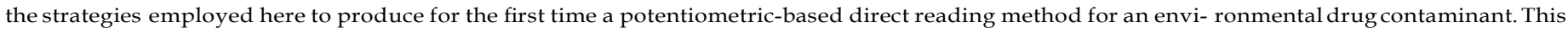
concepthas been applied to sulfamethoxazole(SMX), oneof the many antibiotics used in aquaculture practices that may occur in environmental waters.

The novel ligand has been produced by imprinting SMX on the surface of graphitic carbon nanos- tructures $(\mathrm{CN})<500 \mathrm{~nm}$. The imprinted carbon

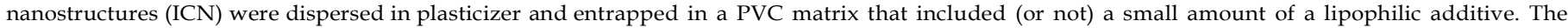

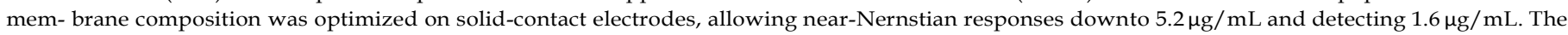
membranes offered good selectivity against most of the ionic compounds in environmental water.

The best membrane cocktail was applied on the smaller end of a $1000 \mu \mathrm{L}$ micropipette tip made of polypropylene. The tip was then filled with

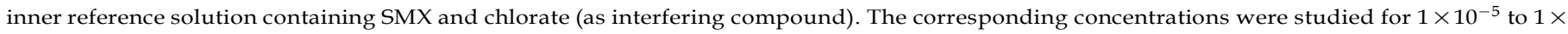
$10^{-10}$ and

$1 \times 10^{-3}$ to $1 \times 10^{-8} \mathrm{~mol} / \mathrm{L}$. The best condition allowed the detection of $5.92 \mathrm{ng} / \mathrm{L}\left(\right.$ or $2.3 \times 10^{-8} \mathrm{~mol} / \mathrm{L}$ )

SMX for a sub-Nernstian slope of $-40.3 \mathrm{mV} /$ decade from $5.0 \times 10^{-8}$ to $2.4 \times 10^{-5} \mathrm{~mol} / \mathrm{L}$.

The described sensors were found promising devices for field applications. The good selectivity of the sensory materials together with a carefully

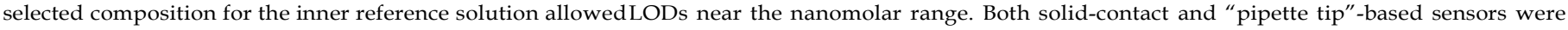
successfully applied to the analysis of aquaculture waters.

\section{Keywords:}

Antibiotic contaminants, Low detection limit, Potentiometry, Solid-contact electrodes, Plastic antibodies

\section{Introduction}

Analytical devices are becoming more and more sophisticated within time, being capable of reaching amazing low detection levels. However, this growing sophistication often increases the price and maintenance expenses around the analytical applications. Moreover, it obstructs portability, which is an emerging requirement among the analytical field. Most controls should be carried on-site and, ideally, via wireless connections.
Considering mainly portability and low-cost, ion-selective electrodes (ISEs) are by far one of the most attractive devices for field applications. The most disseminated way to prepare such detectors is to use a polymeric membrane as active layer consisting of PVC matrix carrying an appropriate ligand and eventually other additional ingredients. Trace level monitoring seems feasible after decreasing the relatively high LODs, typically lying within the micromolar range, and their associated limited selectivity.

The response of most membrane ISEs may be attributed to iontransfer processes across the membrane/sample solution interfaces. However, the ion flux across the bulk membrane affects the analyte activity at this interface (Amemiya, 2007). The analyte ions leaching from the membrane may reach very high levels at the phase boundary when the concentration of primary ions in the sample solution is very low, which in turn hinders the LODs. Forcing a flux of analyte ions toward the inner solution has been 
given as a simple way to prevent this leaching and lowering LODs (Sokalski et al., 1997; Amemiya, 2007). Numerous parameters may be adjusted for this purpose, including the composition of the inner electrolyte and the selective membrane (Sokalski et al., 1999b).

The concentration of the primary ion in the inner compartment should be set to a low value, in order to generate a net flux of primary ions towards this side of the membrane. The exact concentration required for this purpose must be studied, because if a high flux is generated, super-Nernstian responses at intermediate concentrations and insensitivity at very low concentrations are expected (Michalska et al., 2004). Moreover, the ion-exchange with interfering ions at the inner membrane side becomes important under this limiting condition, supporting the need of an interfering compound inside the inner compartment (Bakker et al., 2004). This concept has been proven feasible to inorganic ionic species but never tested in organic trace levels.

Lowering LODs is only possible as long as the ISE shows good selectivity for the target ion (Sokalski et al., 1999b). In general, the selectivity of an ISE is reached by doping the membranes with an ionophore or neutral/charged carrier establishing selective and reversible complexes with the target analyte (Amemiya, 2007). In essence, a good ligand should selectively bind the analyte and remain in the membrane phase. Molecular imprinting polymers meet these criteria because they consist on synthetic robust network materials carrying specific binding sites for the imprinted molecules. These materials have been introduced successfully as potentiometric ionophores in PVC membranes for detecting pesticides (Kamel et al., 2008), antibiotics (Moreira et al., 2010) or proteins (Moreira et al., 2011). The first and second works use bulk imprinting and confirmed that the conventional ionophores could be successfully replaced by these new man-tailored polymers, expected to carry out host-guest interactions. The last work employed surface-imprinting, ensuring a layer-by-layer control. This approach was used because protein imprinting has many difficulties associated to the many conformational changes of the target analyte (Moreira et al., 2011). This technique anchored the protein, diminishing the number of its possible orientations on the sensory surface and offering a higher number of binding sites per contact area.

The above concepts have been merged to develop a simple and low-cost device for on-site detection of water organic contaminants. In general, water matrices are regularly contaminated with antibiotics, pesticides, herbicides, hormones, pigments, minerals, and vitamins (Goldberg et al., 2001; JSA, 2007). Antibiotics are here a major concern because they have been associated to many emerging cases of resistant bacteria (Jones et al., 2001). Thus, sulphamethoxazole (SMX) has been selected as test compound. It is a sulphonamide antibiotic drug for human or veterinary use (Brackett et al., 2004; Strom et al., 2003) that has been detected in environmental waters (García-Galán et al., 2011), coming from domestic or industrial sectors, including aquaculture activities. It shows potential toxicity to aquatic organisms and has also been related to resistant bacteria episodes (Jones et al., 2004). Its determination has been conducted by liquid-chromatography (LC) coupled with mass spectrometry (MS), UV, fluorescence or electrochemical detection, gas-chromatography (GC)/MS, capillary electrophoresis (CE), and immunochemical assays (Cai et al., 2008; He et al., 2008; Stoev and Michailova, 2000; Fuh and Chu, 2003; Vanesa et al., 2010). All these are too expensive or unable to carry out on-site analysis. The potentiometric sensors described so far donothave the necessary LOD for field applications (Almeida etal., 2011, 2012).

In this work, a novel ligand compound for SMX was designed by carrying out for the first time surface imprinting on carbon nanostructures $(\mathrm{CN})$. This material was tested first as a potentiometric ionophore in PVC membranes, displaying suitable selective character for the target ion. After that, the electrolyte composition of an electrode body consisting of a pipette tip was optimized and used to carry out steady state analyses of waters from different aquaculture facilities.

\section{Experimental}

\subsection{Equipment}

Emf in steady-state assays was measured by a Crison $\mathrm{pH}$-meter GLP 21 ( $\pm 0.1 \mathrm{mV}$ sensitivity), at room temperature, and under constant stirring. The reference electrode was a Crison, 5240, of double-junction. When necessary, the $\mathrm{pH}$ was measured by a Crison GLP 21 combined glass electrode connected to a Crison $\mathrm{pH}$ meter. An SBS vortex, MVOR 03, was used to grant the good mixing of the reacting solutions. Insoluble materials were suspended in a Sonorex digitec sonicater. Infrared spectra were collected by a Nicolet iS10 FTIR spectrometer (Thermo Scientific), coupled to an ATR (Attenuated Total Reflectance) sampling accessory of diamond contact crystal, also from Nicolet. Raman experiments were performed using a JOBIN-YVON LABRAM spectrometer. A scanning electron microscope JEOL JSM 35C/Noran Voyager, was used.

\subsection{Reagents and solutions}

All chemicals were of analytical grade and deionised water (conductivity $<0.054 \mu \mathrm{S} / \mathrm{cm}$ at $25^{\circ} \mathrm{C}$ ) was used. SMX, 3-aminophenylboronic acid (APBA), tris(hydroxymethyl) aminomethane (TRIS), graphite (nanopowder $<500 \mathrm{~nm}$ and mean pore size of $137 \mathrm{~A}^{\circ}$ ), $N$-ethyl- $N^{\prime}$-(3-dimethylaminopropyl) carbodiimide hydrochloride (EDAC), tetraoctylammonium bromide (TOABr) and 4-(2-hydroxyethyl)-1-piperazineethanesulfonic acid (HEPES) were purchased from Sigma-Aldrich. o-Nitrophenyloctyl ether (oNPOE), poly (vinyl chloride) (PVC) of high molecular weight, sodium persulphate (SPS), and $N$-hydroxysuccinimide (NHS) were obtained from Fluka. Tetrahydrofuran (THF) was purchased to Riedel-deHäen and sulphuric acid and nitric acid to Merck.

Working SMX solutions ranged from $1 \times 10^{-10}$ to $1 \times$ $10^{-5} \mathrm{~mol} / \mathrm{L}$. They were prepared by accurate dilution of a stock solution of $1 \times 10^{-4} \mathrm{~mol} / \mathrm{L}$ in $1 \times 10^{-4} \mathrm{~mol} / \mathrm{L}$ HEPES buffer, $\mathrm{pH} 5.1$.

\subsection{Synthesis of imprinted carbon nanostructures}

About 200mg of graphitic carbon nanostructures (CN) were suspended in a mixture of concentrated sulphuric acid and nitric acid $(3: 1, v / v)$, and sonicated for $2 \mathrm{~h}$. The nanostructures were then filtrated by a $0.45 \mu$ m nylon membrane, washed with deionized water until the wash out solution had no acid character and dried at room temperature under a nitrogen atmosphere for $12 \mathrm{~h}$.

About $100 \mathrm{mg}$ of oxidized $\mathrm{CN}$ were dispersed in $5.0 \mathrm{ml}$ of deionized water by $1 \mathrm{~min}$ sonication. Then, $2.0 \mathrm{ml}$ of a $0.05 \mathrm{~mol} / \mathrm{L} \mathrm{HEPES}$ buffer solution $(\mathrm{pH} \sim 5.1)$ and $5.0 \mathrm{ml}$ of a $50 \mathrm{mg} / \mathrm{ml} \mathrm{NHS}$ aqueous solution were added to the above suspension. $1.5 \mathrm{ml}$ of fresh EDAC aqueous solution $(10 \mathrm{mg} / \mathrm{ml})$ was added quickly, and the mixture was continually stirred at room temperature for $30 \mathrm{~min}$. The suspension was then filtered and rinsed thoroughly with a $0.005 \mathrm{~mol} / \mathrm{L}$ HEPES buffer solution to remove excess EDAC and NHS.

Reactive CN were re-dispersed in $9.0 \mathrm{ml}$ of $0.005 \mathrm{~mol} / \mathrm{L} \mathrm{HEPES}$ buffer and $1.0 \mathrm{ml}$ of $1 \mathrm{mg} / \mathrm{ml} \mathrm{SMX}$ solution. The nanostructures were then incubated with $1 \mathrm{~mol} / \mathrm{L}$ TRIS, washed with deionised water and finally added of $1.0 \mathrm{~mL}$ of $0.05 \mathrm{~mol} / \mathrm{L}$ ABPA solution. After $45 \mathrm{~min}, 1.0 \mathrm{~mL}$ of $0.025 \mathrm{~mol} / \mathrm{L}$ SPS solution was added. The subsequent polymerisation was carried out at room temperature for $1 \mathrm{~h}$, and the modified $\mathrm{CN}$ were washed again with deionised water. Finally, a diluted acid solution was added to break the amide bond 
binding the template to the modified material. This was made at room temperature for $12 \mathrm{~h}$. The imprinted carbonnanostructures (ICN) were then filtered, thoroughly washed with water and dried.

Non-imprinted carbon nanostructures (NICN) materials were also prepared by following the same steps and excluding the template from the procedure.

\subsection{Membrane composition and ISE constructions}

SMX selective electrode membranes were prepared by dissolving $15 \mathrm{mg}$ of ICN and $0.4 \mathrm{mg}$ of TOABr, together in PVC and $o$ NPOE $(1: 3(w / w))$ to give a total cocktail mass of $500 \mathrm{mg}$. This solution was then mixed with $2.0 \mathrm{~mL}$ of THF.

The ICN was tested as potentiometric ionophore in PVC membranes casted over a solid-state contact. This conductive support was prepared by mixing Araldite Mand hardener in a 2.5:1.0 (w/w) ratio. This epoxy resin was then mixed with graphite powder in a $2: 1(\mathrm{w} / \mathrm{w})$ ratio and applied at $0.2 \mathrm{~cm}$ of the end of the electrode body made of Perspex tube (external ø $12 \mathrm{~mm}$, internal $\varnothing 10 \mathrm{~mm}$, length $15 \mathrm{~cm}$ ).

ISEs of internal referencesolutionwere used toreachlower LOD. The electrode body consisted on pipette tips of $1000 \mu \mathrm{L}$. These were dipped into the membrane solution until the column of the liquid reached $\sim 4 \mathrm{~mm}$. Then, the pipettes were placed in a holder and the THF wasleft to evaporateovernight, yielding solvent polymeric membranes located at the end of the pipette tip. An Ag wire with a thin layer of $\mathrm{AgCl}$ was dipped in the internal electrolyte and used as internal reference material.

A shielded electrical wire was employed to connect the ISEs to the commutation unit. The remaining conducting surfaces were isolated with heat shrink sleeve. The free end of the cable waslinked to BNC (BayonetNutCoupling) terminal.

\subsection{Potential measurements}

All readings were conducted at room temperature $\left(19-21^{\circ} \mathrm{C}\right)$ and in stirred solutions. The assembly of the potentiometric cell was $\mathrm{Ag}, \mathrm{AgCl}(\mathrm{s})|\mathrm{KCl}, 1 \mathrm{~mol} / \mathrm{L}| \mid$ buffered samplesolution $\left(1 \times 10^{-4} \mathrm{~mol} / \mathrm{L}\right.$ HEPES) | SMX selective membrane | inner electrolyte | $\mathrm{AgCl}$ (s), Ag. The inner electrolyte had SMX and/or chlorate, of variable concentrations. The electrodes were conditioned in $1 \times 10^{-8} \mathrm{~mol} / \mathrm{L} \mathrm{SMX}$ to minimize the effect of co-extraction from the sample side during conditioning.

The electrochemical operating characteristics of the ISEs were assessed by calibration curves $(n=3)$ (Buck and Cosofret, 1993) following IUPAC recommendations (Buck and Lindner, 1994). These were conducted in HEPES buffer, $1 \times 10^{-4} \mathrm{M}$, for increasing SMX concentrations. Theemf was recorded after stabilization to $\pm 0.2 \mathrm{mV}$ and plotted as a function of logarithm SMX concentration.

Selectivity studies followed the matched potential method (MPM) for background SMX solutions of $8.0 \times 10^{-5} \mathrm{~mol} / \mathrm{L}$ that were increased up to $1.4 \times 10^{-4} \mathrm{~mol} / \mathrm{L}$.

The coefficient of variation $\left(\mathrm{Cv}_{\mathrm{w}}\right)$ was the ratio of the standard deviation to the mean. The within-day variability was assessed by measuring the closeness of agreement between 5 readings of a known standard concentration during the same day. The betweenday variability was assessed similarly but in 5 different days. The concentrations of SMX were $1.3 \times 10^{-4}$ and $5.9 \times 10^{-6} \mathrm{~mol} / \mathrm{L}$ for electrodes of solid contact and with inner reference solution, respectively. The percentage recovery was calculated for theseconcentrations.

\subsection{FTIR and Raman analysis}

The chemical modification of the $\mathrm{CN}$ was followed by FTIR analysis. Theinfrared spectrawerecollected after background correction.
The number of scans was 32 for both sample and background. $X$ axis was wavenumber, ranging $500-4000 \mathrm{~cm}^{-1}$, and $Y$-axis was $\%$ transmittance. Resolution was 4000 .

Raman experiments were conducted with a He-Ne laser of monochromatic red light with $632.8 \mathrm{~nm}$ and $20 \mathrm{~mW}$. Raman spectra were measured with a density filter to avoid thermal decomposition of samples by the laser. A $50 \times$ air objective lens of an Olympus optical microscope was used to focus the laser beam on the sample and also to collect the scattered radiation. A highly sensitive CCD camera was used to collect the Raman spectra. Extended scans from 100 to $3500 \mathrm{~cm}^{-1}$ in several sampling points were performed on each sample. All samples were deposited in powder form on a glass slide, without using any solvent.

\subsection{Analysis of aquaculture waters}

Aquaculture water samples were obtained in several points of sweet water aquaculture units in the Douro River. All samples were collected in duplicate to amber glass bottles, with polypropylene open-top screw caps and Teflon-silicon septa. The samples were labelled immediately after collection and kept chilled in thelaboratory.

The samples had no antibiotic and were spiked with SMX up to 18,100 or $181 \mathrm{ng} / \mathrm{L}$ when the analysis was conducted by ISEs of solid or liquid-contact, respectively. The analysis was carried out immediately after calibration of the potentiometric units.

\section{Results and discussion}

\subsection{Imprinting on carbon nanostructures}

Graphitic CN are relatively inexpensive materials, displaying suitable features for application in electrical devices. It consists of layers of $\mathrm{sp}^{2}$ hybridized carbon atoms, mostly arranged ina hexagonal lattice with a distance between planes of $0.335 \mathrm{~nm}$. These layers are not evident under regular scanning electron microscopy and carry a random packing mostly in hexagonal form, as may be seen in Fig. S1.

CN may act as a physical support to the imprinted layer, providing extensive surface area for carrying out surface imprinting, and thus allowing a nanostructured controlled process. The overall process of SMX surface imprinting on $\mathrm{CN}$ may be found in Fig. 1. The $\mathrm{CN}$ were first oxidized to ensure carboxylic acid groups on its surface. These groups were then activated by EDAC and added of NHS to form a stable ester. The resulting ester reacted by nucleophilic substitution with the amine group on SMX, linking the CN materials to the target analyte by an amide bond. TRIS was used next to block carboxylic acid functions that remained active. It carries an amine group that reacts with the active carboxylic acid producing an amide. The resulting $\mathrm{CN}$ were then let stand in APBA to let organize the monomers around the SMX structure through electrostatic interactions. In addition, the vicinal hydroxyl functions coming from the TRIS layer were able to react with the boronic acid function in the APBA monomers, binding these to the $\mathrm{CN}$. These bound monomers were subsequently able to participate in the polymerization reaction taking place after, when more APBA and initiator are added, binding the imprinting layer to the $\mathrm{CN}$. This was indeed the imprinting stage. The imprinted sites were obtained by removing SMX from the structure and these sites should be able to rebind again to another molecule of SMX.

At a first glance, this may seem a similar process to that presented by Almeida et al. (2011). However, the chemical path follows a completely different scheme, accounting for the great chemical differences between the silica spheres employed there and the $\mathrm{CN}$ used here. Overall, the silica spheres were linked to 


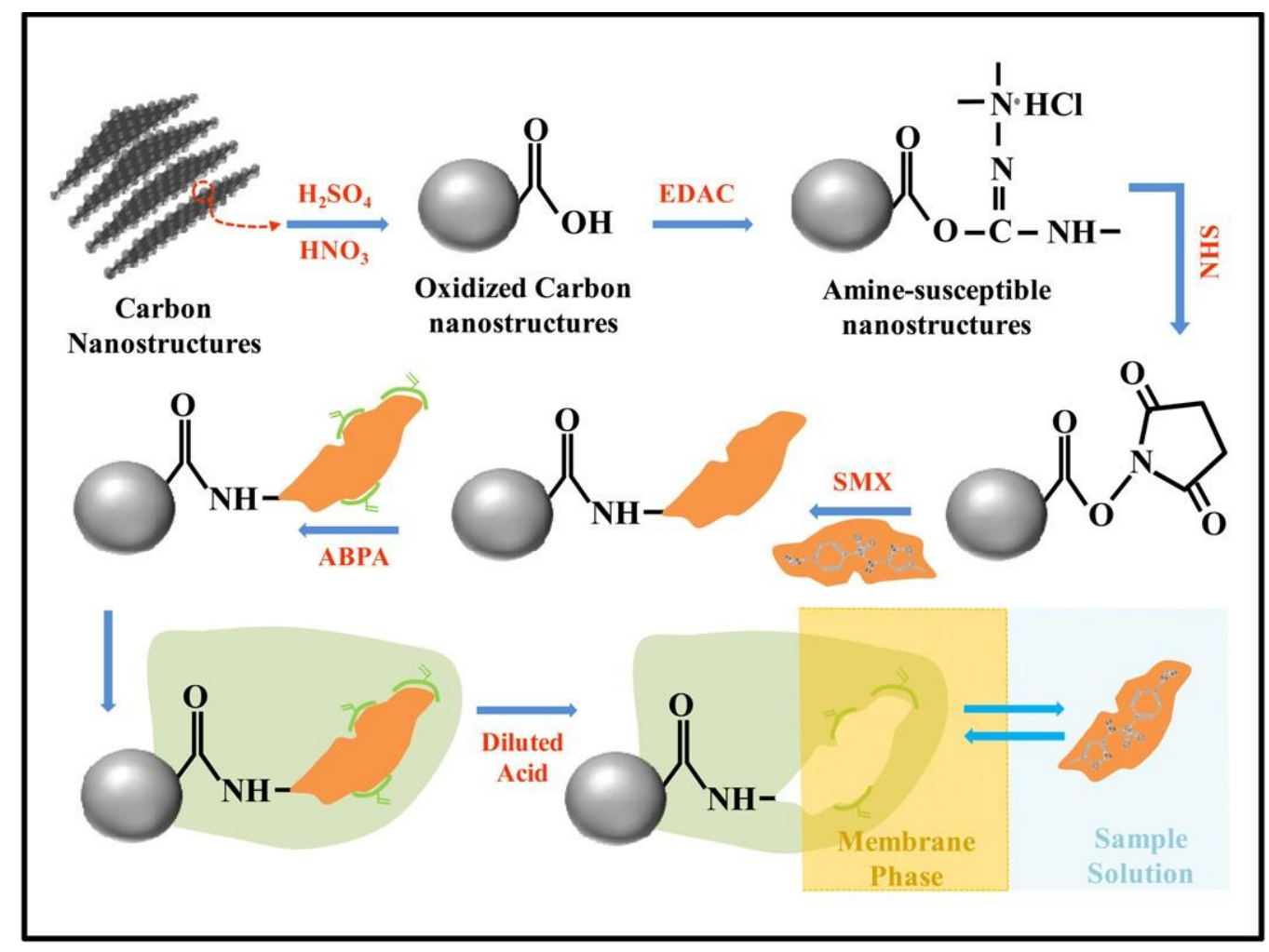

Fig. 1. Design of the plastic antibodies over graphitic carbon nanostructures.

sol-gel materials and modified with a glutaraldehyde layer to enable introducing the protein on top of it, while the graphitic carbon nanostructures were oxidized and the carboxylic groups activated by EDAC/NHS prior to protein binding. Comparing these two imprinting schemes, this last one turns out more effective because the imprinting layer is constructed immediately over the oxidized carbon material and has less monolayers. Evidently, each additional monolayer is an additional critical step to the overall procedure.

All modification steps were followed by FTIR experiments. The spectra of bare, oxidized, imprinted and non-imprinted $\mathrm{CN}$ are presented in Fig. 2(top). In general, the oxidation of the carbon material to carboxylic acid groups was confirmed by the appearance of broad and small absorption band near $3300 \mathrm{~cm}^{-1}$. The small absorption peak at $1694 \mathrm{~cm}^{-1}$ corroborated with this observation, being attributed to the stretch of the double bond on the carbonyl group. The presence of the polymeric layer (both in ICN and NICN) was confirmed by the large increase of the broad band centered at $\sim 3200 \mathrm{~cm}^{-1}$. This band has been attributed to the $\mathrm{O}-\mathrm{H}$ stretching motions of the $\mathrm{B}(\mathrm{OH})_{2}$ of the APBA (Peak et al., 2003). It may also account for the stretching of $\mathrm{NH}$ bonds in the synthetic polymer. The peaks at 1034.0 and $1207.5 \mathrm{~cm}^{-1}$ are present only in ICN and NICN materials, showing the plane bending of B-O $\mathrm{H}$ and/or the $\mathrm{C}-\mathrm{N}$ stretch. No differences could be observed on the FTIR spectra of ICN and NICN materials, because they differed only on the spatial arrangement of the molecular structure of the outer layer and presented the same chemical composition.

Electron microscopy observation was also tried out(Fig.S1). The graphitic nanostructures and their chemically modified materials were however too small to be observed either by scanning or transmission electron microscopes. The transmission images showed a different packing for the $\mathrm{CN}$, but this could not be attributed to a successful modification of the ICN/NICN forms, because the observed matrix was heterogeneous and this pattern was not regular. Still, their chemical modification seemed clear by the different behaviours of these materials when they were suspended for observation under transmission: while $\mathrm{CN}$ dispersed readily in chloroform, ICN and NICN dispersed immediately in ethanol but deposited in the tube when chloroform was used.

Raman scattering analysis was also tried out, searching for the structural differences within $\mathrm{CN}, \mathrm{ICN}$ and NICN materials. The obtained Raman spectra are shown in Fig. 2(bottom). The spectra of graphitic CN showed three main peaks at 1332.7, 1577.1 and $2665.9 \mathrm{~cm}^{-1}$ (Table S1), assigned to the D, G and 2D bands of graphite (Fig. 2). The G Peak represented $\mathrm{sp}^{2}$ bonds (planar configurations) and is inherent in graphite lattices. The $\mathrm{D}$ band was induced from a decrease of the domain size of graphite crystals (Sun et al., 1999), showing the introduction of the $\mathrm{sp}^{3}$-type structural disorder. The small shoulder in $\mathrm{G}$ band was identified as $\mathrm{D}_{2}$ band and, in conjunction with $\mathrm{D}$, shows well crystallized graphite (Wopenka and Pasteris, 1993). The band at $2665.9 \mathrm{~cm}^{-1}$ has been assigned to the 2D band (Qian et al., 2009).

ICN and NICN materials showed the same D and G bands as the $\mathrm{CN}$ (Table S1). These bands are however much broader than those of the CN (Fig. 2), indicating the existence of mainly amorphous carbon, where more phonons are allowed and broadening the discrete spectral lines is expected. The relative intensity of D and G bands was also obviously changed, with $\mathrm{G}$ band becoming greater than D band (Table S1). This observation corroborated well with the presence of a polymeric layer on the overall structure, which increased the $\mathrm{sp}^{3}$ hybridization bonding. Furthermore, these bands appear with a significant shift to the CN. While the D band shifted to -2.8 and to $+1.2 \mathrm{~cm}^{-1}$ in ICN and NICN materials, respectively, the $G$ band shifted $+17.2 \mathrm{~cm}^{-1}$ in both materials. In general, these shifts have been attributed to the structural changes introduced on the material organization by growing a polymeric layer over the $\mathrm{CN}$. Furthermore, the D bands from ICN and NICN were shifted by $4 \mathrm{~cm}^{-1}$, thus confirming the existence of minor structural changes 

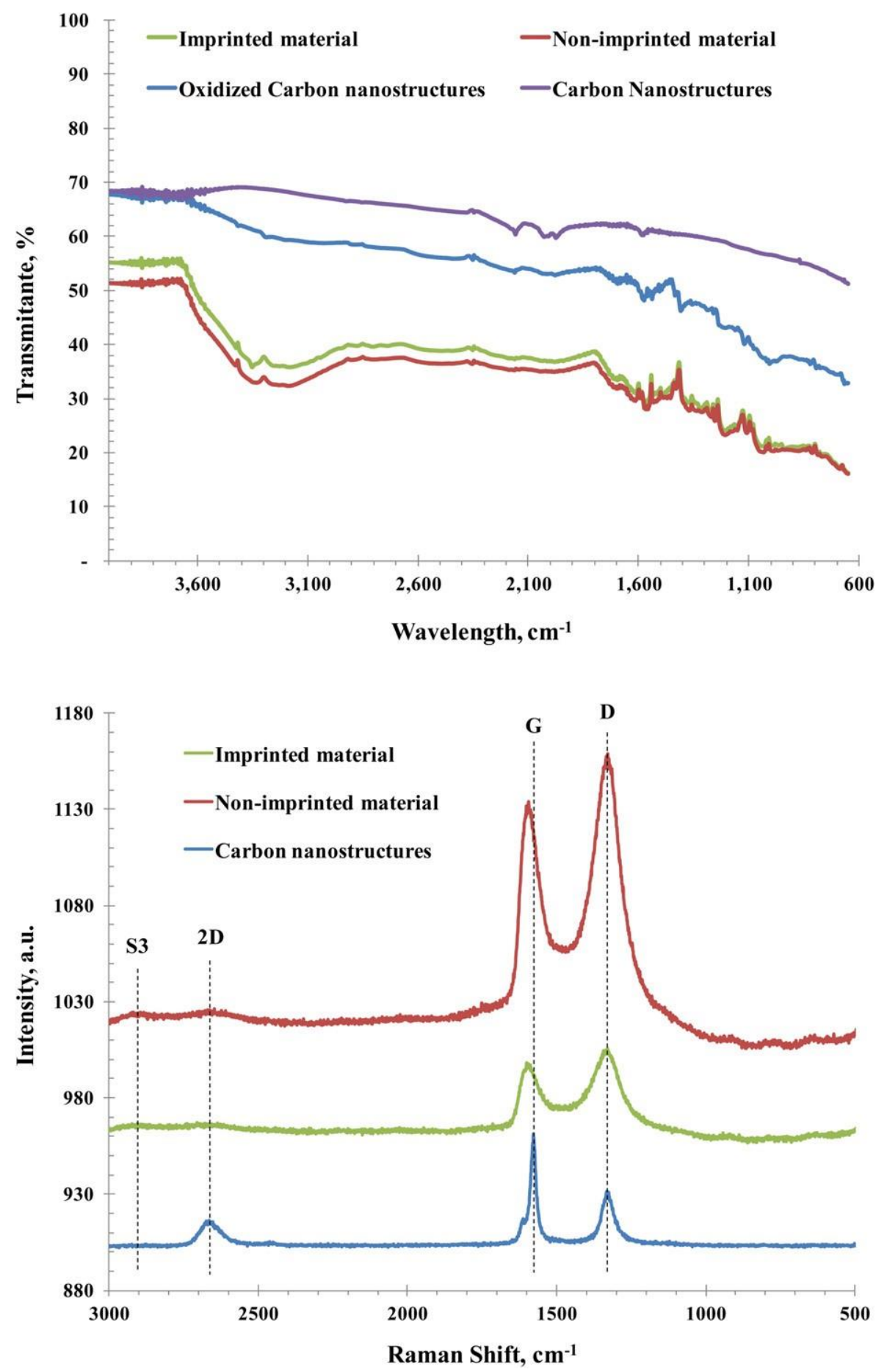

Fig. 2. FTIR (top) and Raman (bottom) spectra of bare, oxidised, imprinted and non-imprinted CN.

between these materials. These changes corroborated also with the differences in 2D and S3 bands (Fig. 2). Overall, the obtained results confirmed the existence of slight structural differences between ICN and NICN materials and major differences between these and the $\mathrm{CN}$.

\subsection{Selective membrane composition}

\subsubsection{General components}

The above materials were used as ionophores for the potentiometric transduction of SMX on PVC membrane ISEs. The selective sensors were prepared by dispersing the active ingredients (ICN or NICN) in a high dielectric constant plasticizer and PVC. A cationic additive was also added to some of the membranes (Table 1), selected according to previous experiments (Almeida et al., 2012).

All the membranes were applied over a solid-contact conductive material made of graphite and epoxy resin. The best composition was set after varying the amount of cationic additive and electroactive material, and checking the analytical performance of the corresponding devices by means of consecutive and periodic calibrations. These studies were conducted for SMX concentrations ranging from $2 \times 10^{-5}$ to $2 \times 10^{-3} \mathrm{~mol} / \mathrm{L}$ (Table 1). 
Table 1

Membrane composition of SMX sensors evaluated in solid contact electrodes and their potentiometric features in $1 \times 10^{-4}$ mol/L HEPES buffer, $\mathrm{pH} 5.1$.

\begin{tabular}{|c|c|c|c|c|c|c|c|c|c|}
\hline Parameter & ISE I & ISE II & ISE III & ISE IV & ISE V & ISE VI & ISE VII & ISE VIII & ISE IX \\
\hline SMX ligand (mg) & $\operatorname{ICN}(5)$ & $\operatorname{ICN}(15)$ & ICN (30) & $\operatorname{ICN}(15)$ & NICN (15) & NICN (15) & - & - & - \\
\hline Additive (mg) & $\operatorname{TOABr}(0.2)$ & $\operatorname{TOABr}(0.2)$ & $\operatorname{TOABr}(0.2)$ & - & $\operatorname{TOABr}(0.2)$ & - & $\operatorname{TOABr}(0.2)$ & TOABr (0.5) & TOABr (1.0) \\
\hline Slope, $\mathrm{mV} /$ decade & $-45.8( \pm 1.3)$ & $-52.5( \pm 0.4)$ & $-48.4( \pm 1.1)$ & $-39.3( \pm 0.6)$ & $-38.7( \pm 0.5)$ & $-27.1( \pm 2.9)$ & $-30.5( \pm 1.6)$ & $-32.2( \pm 1.0)$ & $-35.4( \pm 0.5)$ \\
\hline$R^{2}(n=3)$ & 0.993 & 0.997 & 0.994 & 0.995 & 0.994 & 0.997 & 0.996 & 0.995 & 0.997 \\
\hline LLLR,ng/mL & $10.4 \times 10^{3}$ & $5.18 \times 10^{3}$ & $5.18 \times 10^{3}$ & $5.19 \times 10^{3}$ & $8.64 \times 10^{3}$ & $10.4 \times 10^{3}$ & $33.2 \times 10^{3}$ & $17.9 \times 10^{3}$ & $15.3 \times 10^{3}$ \\
\hline $\mathrm{LOD}, \mathrm{ng} / \mathrm{mL}$ & $3.10 \times 10^{3}$ & $1.57 \times 10^{3}$ & $1.61 \times 10^{3}$ & $1.57 \times 10^{3}$ & $2.62 \times 10^{3}$ & $3.14 \times 10^{3}$ & $10.1 \times 10^{3}$ & $5.43 \times 10^{3}$ & $4.64 \times 10^{3}$ \\
\hline $\mathrm{Cv}_{\mathrm{W}}(\%)$ & 2.83 & 0.796 & 2.27 & 1.44 & 1.42 & 10.7 & 5.15 & 3.12 & 1.55 \\
\hline Within-day variability, \% & 1.35 & 1.01 & 1.76 & 2.98 & 2.08 & 0.639 & 2.47 & 0.283 & 1.13 \\
\hline Between-day variability, \% & 1.58 & 1.22 & 2.21 & 2.67 & 2.71 & 1.11 & 1.79 & 0.231 & 0.954 \\
\hline Recovery, \% & 95.2 & 99.3 & 97.3 & 98.0 & 98.7 & 90.3 & 92.7 & 100.8 & 96.7 \\
\hline
\end{tabular}

LOD: limit of detection, LLLR: lower limit of linear range; $R^{2}$ : squared correlation coefficient; RSD: relative standard deviation; $\mathrm{Cv}_{\mathrm{w}}$ : reproducibility;

\subsubsection{Cationic additive}

The cationic additive was employed to improve the permselectivity of the membranes, thus favouring the electrode performance. However, it was important to check that the observed behaviour could be attributed mostly to the novel designed ligand and not to the additiveitself. For this purpose, several ISEs were tested having only additive (VII-IX) in different minimal amounts. The results pointed out that the additive was unable to provide a Nernstian response. The lower amounts of additive decreased the sensitivity of the response, but widened the linear concentration range. Thus, an amount of $0.2 \mathrm{mg}$ of additive was selected to test the behaviour of both ICN and NICN materials.

In general, the response of the electrodes pointed out that the additive was essential to reach an acceptable slope, especially for ISEs with ICN materials. The addition of this compound, even in a very low amount, resulted in a significant slope increase, around $40 \%$ (comparing ISE IV with II and VI with V). This behaviour was attributed to the existence of neutral-ionophore material: the anionic analyte in the membrane formed negatively charged structures with the neutral ionophore SMX receptor sites (Fig. 1), thus requiring the additional incorporation of lipophilic ions of opposite charge to ensure permselectivity. The anionic SMX was bound to the imprinted sites most probably by hydrogen bond formation with the amine or borate groups of the imprinted layer.

\subsubsection{Amount of SMX ligand}

Nernstian responses are obtained when the primary ion is the only major ion being transferred between samples and membrane phases (Amemiya, 2007) and the free primary ion activity in the membrane is buffered by its receptor (Antonisse and Reinhoudt, 1999). For neutral anion receptors, this constant ion activity is achieved by ensuring a constant ratio between the concentration of the anion-receptor complex and the free receptor, which in turn depends on the amount of lipophilic positive sites added to the membrane to compensate the charge of the complexed anions (Antonisse and Reinhoudt, 1999). In the present study, the ratio of cationic additive/receptor site is impossible to calculate, because the chemically modified $\mathrm{CN}$ are polymeric materials of unknown molar mass per number of binding sites.

Thus, no molar ratio may be established and the best amount of ionophore can only be found by experimental testing. The amount of electroactive material acting as SMX ligand added to the membrane ranged from 5 to $30 \mathrm{mg}$ (ISEs I to III). An amount of $15 \mathrm{mg}$ was the one leading to the highest slopes. The other conditions tested gavelower sensitivities, displaying a slope decrease of about 13 and $8 \%$, respectively. The linear concentration ranges were narrower only for the minimum amount tested. So, the ISEs used in further experiments included $15 \mathrm{mg}$ of ICN. Overall, the time taken by the electrodes to reach a steady potential $( \pm 1 \mathrm{mV})$ was small, always less than $30 \mathrm{~s}$, even for the highest concentrations tested.

\subsection{Imprinting effect}

The imprinting effect could be observed by comparing ISEs differing only on this membrane ingredient: ISEs II with V and ISEs IV with VI. The main differences displayed by each pair of ISEs regarded mostly slope, limit of detection and linear range. All membranes relying on imprinted materials showed much higher slopes and lower limits of detection than those with non-imprinted ones, as may be seen in Table 1 . The enhanced performance of the imprinted-based devices may be therefore attributed to the ability of carrying out stereochemical recognition of the analyte. The small response observed for NICN was attributed tonon-specific electrostatic interactions between SMX and this material.

\subsection{Effect of $p H$}

Theeffect of $\mathrm{pH}$ on the response of the ISEs was studied by checking the emf of a $1 \times 10^{-2} \mathrm{~mol} / \mathrm{L}$ SMX solution over the $\mathrm{pH}$ range of 3-10. Each $\mathrm{pH}$ tested was obtained by adding small amounts of concentrated sulphuric acid or saturated sodium hydroxide solutions. In general, the potential remained within $\pm 5 \mathrm{mV}$ from $\mathrm{pH}$ 5 to 8 . Increasing $\mathrm{pHs}$ gave decreasing emfs that were attributed to hydroxyl interference. Decreasing $\mathrm{pHs}$ gave also rise to decreasing emfs, this time attributed to a chloride interference, occurring simultaneously with the deprotonation of SMX.

This operational $\mathrm{pH}$ range was confirmed later by calibrating ISEs II and V in pHs 4.0, 5.1, 6.5 or 9.0. As expected, the more extreme conditions were outside the above range. The analytical performance was completely opposite for $\mathrm{pHs} 4.0$ and 9.0, with ISEs changing from super-Nernstian (104 mV/decade) to sub-Nernstian ( $44 \mathrm{mV} /$ decade) behaviour and the detection limits decreasing from 63.8 to $3.29 \mu \mathrm{g} / \mathrm{mL}$, respectively. The $\mathrm{pH}$ condition displaying a closest behaviour to that expected by the Nernst equation was $\mathrm{pH}$ 5.1. This was the one selected for further studies.

\subsection{Sensor selectivity}

Selectivity studies followed the matched potential method (MPM) and were conducted against $\mathrm{PO}_{4}{ }^{2-}, \mathrm{CH}_{3} \mathrm{COO}^{-}, \mathrm{BO}_{3}{ }^{3-}, \mathrm{CN}^{-}$, $\mathrm{SO}_{4}{ }^{2-}, \mathrm{F}^{-}, \mathrm{CO}_{3}{ }^{2-}, \mathrm{Cl}^{-}, \mathrm{HCO}_{3}{ }^{-}, \mathrm{NO}_{3}{ }^{-}, \mathrm{NO}_{2}{ }^{-}, \mathrm{ClO}_{3}{ }^{-}$and tartrate. The initial concentration of primary ion was set to $8.0 \times 10^{-5} \mathrm{~mol} / \mathrm{L}$ and the activity of the primary ion solution added into this was $1.4 \times 10^{-4} \mathrm{~mol} / \mathrm{L}$. The average corresponding potential change was about $13 \mathrm{mV}$. The interferences were assessed by adding small aliquots of the corresponding solutions into the primary ion solution of $a_{\mathrm{A}}$ until the same potential change was observed.

Overall, the $\log K^{\mathrm{POT}}$ values ranged from -3.6 to 0.47 , showing negligible interference for most of the observed species. Only chlorate showed a positive $\log K^{\mathrm{POT}}$ and was taken as a severe interference. The relative order of interference was $\mathrm{ClO}_{3}{ }^{-}>\mathrm{NO}_{3}{ }^{-}>\mathrm{NO}_{2}{ }^{-}>$tartrate $\sim \mathrm{CN}^{-}>\mathrm{Cl}^{-}>\mathrm{CH}_{3} \mathrm{COO}^{-}>$ 


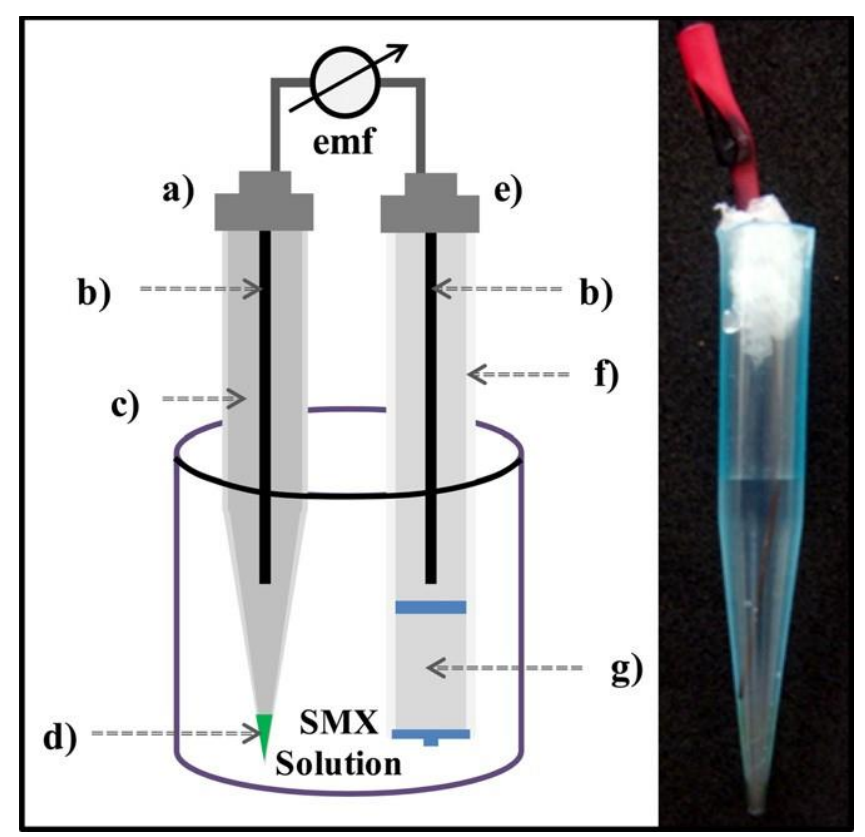

Fig. 3. Schematic diagram of the potentiometric cell. (a) Ion-selective electrode; (b) inner reference ( $\mathrm{Ag} / \mathrm{AgCl})$; (c) inner filling solution; (d) ion-selective membrane; (e) reference electrode; (f) reference electrolyte; (g) bridge electrolyte. (Insert: photography of the pipette-tip electrode.)

$\mathrm{HCO}_{3}{ }^{-}>\mathrm{F}^{-}>\mathrm{SO}_{4}{ }^{2-}>\mathrm{PO}_{4}{ }^{2-}>\mathrm{CO}_{3}{ }^{2-}>\mathrm{BO}_{3}{ }^{3-}$. This sequence deviated from the Hofmeister series, indicating that the mechanism of action was not governed by ion-exchange.

In general terms, the more interfering ion was chlorate. Thus, this specific ion was used as interfering compound in the inner electrolyte of ISEs made from pipette tips.

\subsection{Liquid contact ISES}

\subsubsection{Inner electrolyte composition}

The composition of the inner electrolyte should be set in such a way that the net flux of primary ions should be forced towards the membrane side contacting with this electrolyte. A low activity of primary ion and a sufficiently high activity of an interfering ion should favour this process. Thus, the inner electrolyte composition was tested for SMX concentrations ranging from $1 \times 10^{-3}$ to $1 \times 10^{-10} \mathrm{~mol} / \mathrm{L}$ and chlorate from $1 \times 10^{-4}$ to $1 \times 10^{-8} \mathrm{~mol} / \mathrm{L}$. An electrolyte with just HEPES was also used and tested as control. The general analytical features of such electrodes are indicated in Table 2 and the general set-up may be seen in Fig. 3 .

The concentration of SMX in the inner electrolyte was studied for a solution containing only HEPES with variable amounts of SMX (Table 2). No interfering species was added at this stage. The obtained results pointed out that for concentrations of SMX lower than $1 \times 10^{-8} \mathrm{~mol} /$ Lnosignificant improvement was observed. The lower limit of linear range (LLLR) followed a similar behaviour. The need to add SMX (in a low concentration) to the inner electrolyte was confirmed by checking the analytical performance of electrodes carrying only HEPES (ISEs M, Table 2). These devices presented a similar behaviour to those carrying a higher amount of SMX (ISE A, Table 2).

The conditioning solution was set by evaluating the effect of different concentrations of primary ion upon the LOD of ISED.Concentrations of $1 \times 10^{-6} \mathrm{~mol} / \mathrm{L}$ and $1 \times 10^{-8} \mathrm{~mol} / \mathrm{L}$ were selected for this purpose. The electrodes were let stand in these solutions for at least $16 \mathrm{~h}$. The subsequent calibration curves were carried by washing the electrodes with water, transferring them to HEPES buffer $1 \times 10^{-4} \mathrm{~mol} / \mathrm{L}$ and adding small aliquots of $1 \times 10^{-4} \mathrm{~mol} / \mathrm{L} \mathrm{SMX}$

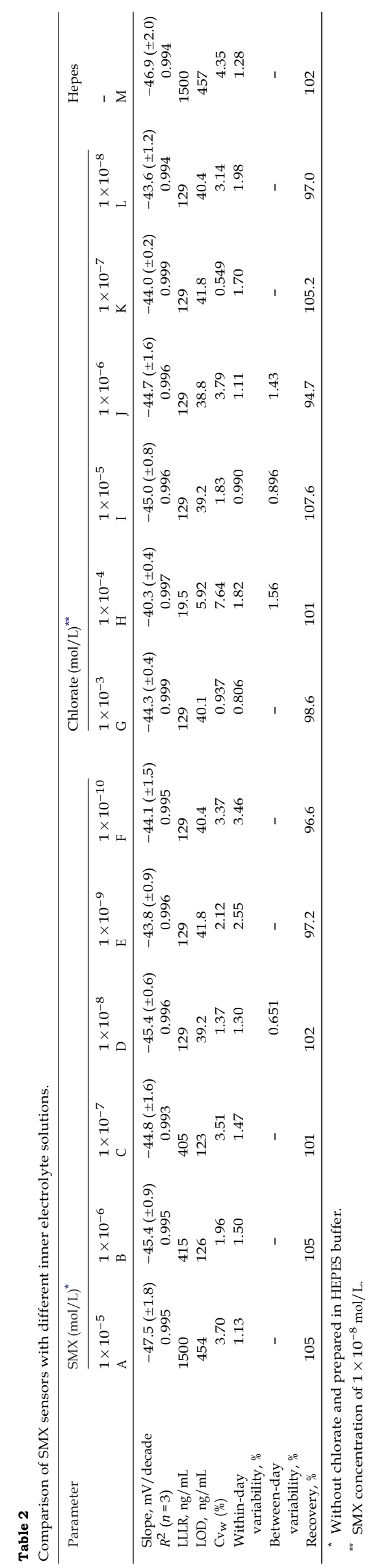


Table 3

Results obtained with solid contact and liquid-contact ISEs in the determination of SMX in aquaculture waters.

\begin{tabular}{|c|c|c|c|c|c|c|}
\hline \multirow[t]{2}{*}{ Sample } & \multicolumn{3}{|c|}{ Solid-contact ISEs } & \multicolumn{3}{|c|}{ ISEs with inner electrolyte solution } \\
\hline & Taken (ng/mL) & Found (ng/mL) & Recovery (\%) & Taken (ng/mL) & Found $(\mathrm{ng} / \mathrm{mL})$ & Recovery (\%) \\
\hline \multirow{2}{*}{1} & $18.1 \times 10^{3}$ & $17.6 \times 10^{3} \pm 0.809 \times 10^{3}$ & 97.5 & 181 & $180 \pm 4.55$ & 99.6 \\
\hline & $100 \times 10^{3}$ & $97.8 \times 10^{3} \pm 2.06 \times 10^{3}$ & 97.8 & 1000 & $1002 \pm 15.5$ & 101.6 \\
\hline \multirow[b]{2}{*}{2} & $18.1 \times 10^{3}$ & $17.9 \times 10^{3} \pm 0.404 \times 10^{3}$ & 99.1 & 181 & $177 \pm 6.8$ & 98.2 \\
\hline & $100 \times 10^{3}$ & $96.6 \times 10^{3} \pm 1.31 \times 10^{3}$ & 96.6 & 1000 & $946 \pm 20.7$ & 94.6 \\
\hline
\end{tabular}

standard solution. The LODs were nearly identical in both conditioning conditions, equal to 39.2 and $39.1 \mathrm{ng} / \mathrm{mL}$, respectively. The conditioning of all electrodes was therefore set to $1 \times 10^{-8} \mathrm{~mol} / \mathrm{L}$, thus avoiding the "saturation" of the ligand sites on the membrane interface when the calibration is carried out at really low SMX concentrations.

The chlorate concentration on the inner electrolyte was tested for ISEs having a fixed amount of SMX $\left(1 \times 10^{-8} \mathrm{~mol} / \mathrm{L}\right)$ and HEPES. The use of a $1 \times 10^{-4} \mathrm{~mol} / \mathrm{L}$ chlorate concentration favoured the electrode performance, leading to a LOD of $2.3 \times 10^{-8} \mathrm{~mol} / \mathrm{L}$. All other conditions tested gavesimilar results. The exact effect of chlorate was identified by preparing additional electrodes with inner electrolyte having only chlorate $\left(1 \times 10^{-8} \mathrm{~mol} / \mathrm{L}\right)$ in HEPES buffer. These electrodes displayed higher LOD than ISE H $(125 \mathrm{ng} / \mathrm{mL})$ and lower slopes $(-38.7 \mathrm{mV} /$ decade), thus confirming the need to include an interfering species in the inner electrolyte.

Overall, the concentration of SMX was set to $1 \times 10^{-8} \mathrm{~mol} / \mathrm{L}$ and the interfering species to $1 \times 10^{-4} \mathrm{~mol} / \mathrm{L}$. The observed behaviour seems to indicate that the LOD is mainly dependent on the interfering species, as long as a sufficiently low concentration of primary ion is ensured. Moreover, a small amount of SMX is necessary for a slight slopeincrease.

\subsection{Analysis of aquaculture waters}

The applicability of the SMX electrodes was tested in aquaculture waters collected on commercial fish farms on the north region of Portugal. The obtained results are summarized in Table 3 and correspond to the mean of at least 3 independent determinations. The standard deviations were always below $5.4 \%$.

A good agreement was found between added and found amounts of the antibiotic. For solid contact electrodes, the results showed recoveries ranging from 96.6 to $99.1 \%$, and for pippette tips electrodes from 94.6 to $102 \%$. The $t$-Student and $F$ tests indicated no significant statistical differences between the means of claimed and potentiometric amounts of both types of electrodes. The calculated $t$ values $(p)$ were 1.2 and 1.5 and the $F$ values were 5.3 and 3.9, for solid contact ISEs and electrodes with inner reference solution, respectively. In both cases, $p$ values were below the tabulated critical value ( $t 1.8$ and 1.9 and $F 6.4$ respectively) for $95 \%$ confidence level, demonstrating that there are no significant differences between claimed and found amounts.

\section{Conclusions}

A new SMXligand was successfully introduced as an ionophore in potentiometric transduction. The imprinting process was effective and used inexpensive material. Improved LODs were enabled by managing the composition of the inner electrolyte with regard to primary and interfering ion concentrations. This was obtained for the first time for an organic anionic species. The devices were coupled to simple construction, low power requirements and low manufacturing costs, relying on pipette tips. The obtained sensors showed suitable analytical performance to carry out on-site analysis.

\section{Acknowledgements}

The authors acknowledge the financial support from FCT, Fundação para a Ciência e Tecnologia, by means of the $\mathrm{PhD}$ grant of SAAA no. SFRH/BD/42509/2007 and the grant no. PEstC/EQB/LA0006/2011.

\section{Appendix A. Supplementary data}

Supplementary data associated with this article can be found, in the online version, at doi:10.1016/j.bios.2012.03.007.

\section{References}

Almeida, S.A.A., Arasa, E., Puyol, M., Martinez-Cisneros, C.S., Alonso-Chamarro, J., Montenegro, M.C.B.S.M., Sales, M.G.F., 2011. Biosens. Bioelectron. 30, 197203.

Almeida, S.A.A., Heitor, A.M., Montenegro, M.C.B.S.M., Sales, M.G.F., 2012. Int. J. Env. Anal. Chem., in press.

Amemiya, S., 2007. In: Zoski, C.G. (Ed.), Chapter 7 in Handbook of Electrochemistry. Elsevier.

Antonisse, M.M.G., Reinhoudt, D.N., 1999. Electroanalysis 11, 1035-1048.

Bakker, E., Bühlmann, P., Pretsch, E., 2004. Talanta 63,3-20.

Brackett, C.C., Singh, H., Block, J.H., 2004. Pharmacotherapy 24, 856-870.

Buck, R.P., Lindner, E., 1994. Pure Appl. Chem. 66, 2527-2536.

Buck, R.P., Cosofret, V.V., 1993. Pure Appl. Chem. 65, 1849-1858.

Cai, Z.X., Zhang, Y., Pan, H.F., Tie, X.W., Ren, Y.P., 2008. J. Chromatogr. A 1200, 144-155.

Fuh, M.R.S., Chu, S.Y., 2003. Anal. Chim. Acta 499, 215-221.

García-Galán, M.J., Díaz-Cruz, M.S., Barceló, D., 2011. Environ. Int. 37, 462-473.

Goldberg, R.J., Elliott, M.S., Naylor, R.L., 2001. Pew Oceans Commission. Arlington, Virginia.

He, J.X., Wang, S., Fang, G.Z., Zhu, H.P., Zhang, Y., 2008. J. Agric. Food Chem. 56, 2919-2925.

Jones, O.A.H., Voulvoulis, N., Lester, J.N., 2001. Environ. Technol. 22, 1383-1394.

Jones, O.A.H., Voulvoulis, N., Lester, J.N., 2004. Crit. Rev. Toxicol. 34, 335-350.

JSA. (Federal Joint Subcommittee on Aquaculture), http://aquanic.org/jsa/wgqaap/, 2007 (assessed 13.10.2011).

Kamel, A.H., Moreira, F.T.C., Almeida, S.A.A., Sales, M.G.F., 2008. Electroanalysis 20, 194-202.

Michalska, A.J., Appaih-Kusi, C., Heng, L.Y., Walkiewicz, S., Hall, E.A.H., 2004. Anal. Chem. 76, 2031-2039.

Moreira, F.T.C., Dutra, R.A.F., Noronha, J.P.C., Sales, M.G.F., 2011. Biosens. Bioelectron. 26, 4760-4766.

Moreira, F.T.C., Kamel, A.H., Guerreiro, J.R.L., Sales, M.G.F., 2010. Biosens. Bioelectron. $26,566-574$.

Peak, A., Luther, G.W., Sparks, D.L., 2003. Geochim. Cosmochim. Acta 67, 2551-2560.

Qian, M., Feng, T., Ding, H., Lin, F., Li, H., Chen, Y., Sun, Z., 2009. Nanotechnology 20, 425702 (6 pp).

Sokalski, T., Zwickl, T., Bakker, E., Pretsch, E., 1999b. Anal. Chem. 73, 1204-1209.

Sokalski, T., Ceresa, A., Zwickl, T., Pretsch, E., 1997. J. Am. Chem. Soc. 119, $11347-11348$.

Sun, Z., Jia, W.B., Shi, X., 1999. Surf Coat. Technol. 122, 277-280.

Stoev, G., Michailova, A., 2000. J. Chromatogr. A 871, 37-42.

Strom, B.L., Schinnar, R., Apter, A.J., Margolis, D.J., Lautenbatch, E., Hennessy, S., Bilker, W.B., Pettitt, D., 2003. N. Engl. J. Med. 349, 1628-1635.

Vanesa, J., Javier, A., Jacinto, G., Pilar, M.M., Ramon, C., 2010. J. Agric. Food Chem. 58, 7526-7531.

Wopenka, B., Pasteris, J.D., 1993. Am. Mineral. 78, 533-557. 